\title{
Plasma generation in an organic molecular gas by an ultraviolet laser pulse
}

\author{
Y. S. Zhang and J. E. Scharer \\ Dopartmont of Electrical and Computer Engineering, University of Wisconsin-Madison, \\ 1500 Johnson Drive, Madison, Wisconsin 53706
}

(Received 31 August 1992; accepted for publication 19 January 1993)

\begin{abstract}
Plasmas are generated in a low ionization potential gas, tetrakis(dimethylamino)ethylene (TMAE) vapor through a one-photon ionization process by an ultraviolet laser beam at a 193 $\mathrm{nm}$ wavelength. The TMAE plasma characteristics are studied by means of a Langmuir probe and microwave scattering. A new method is used to measure the $193 \mathrm{~nm}$ ultraviolet (UV) photon absorption cross section in TMAE. It is determined to be $1.1 \pm 0.3 \times 10^{-17} \mathrm{~cm}^{2}$ from the axial profile of electron density. The temporal evolution of electron temperature is measured. A peak electron density of $n_{e}=5 \times 10^{13} / \mathrm{cm}^{3}$ and peak electron temperature of $T_{e}=1 \mathrm{eV}$ are measured at $500 \mathrm{~m}$ Torr TMAE pressure. The plasma decay process is studied, and the electron-ion recombination coefficient is measured to be $5.4 \pm 0.5 \times 10^{-6} \mathrm{~cm}^{3} / \mathrm{s}$. A theoretical model is derived to describe the photon flux and the one photon ionization process. An application of a TMAE plasma as a mirror for microwave reflections is proposed.
\end{abstract}

\section{INTRODUCTION}

The interaction of electromagnetic waves with a plasma has been a scientific research interest for decades. Recently, there has been a considerable research effort in laboratories to study the use of man-made plasmas to reflect, shield, and absorb electromagnetic waves. ${ }^{1-9}$ These research efforts involve basic physics issues as well as many interesting potential applications. One of these efforts is aimed at generating artificial ionospheric mirrors (AIM) in the lower atmosphere by high power microwave beams to relay communications or reflect the signals for over the horizon radar (OHR). ${ }^{5,6}$ Another potential application is to use a rotating plasma sheet to steer a high frequency radar beam. This is desirable because in a phased-array antenna system, if the radar frequency is high, the number of radiating elements becomes large, and the packing density is high. ${ }^{7,8}$ Another promising application is to use collisional plasmas as wide-band microwave absorbers to shield objects from high power microwave or radar signals. 1,9

In a practical system which uses plasmas to reflect or absorb waves, one often requires a plasma source that is compact, highly efficient, controllable, and produces a desired density profile. Although there are many ways to generate ionization, few plasma sources meet the requirements mentioned above. Plasmas can be generated in a gas of organic molecules by ultraviolet radiation. Woodworth et al. ${ }^{10}$ have studied plasma generation in different low ionization potential organic gases by ultraviolet lasers mainly through a two-photon ionization process. Vidmar proposed the UV photoionization of an organic vapor such as tetrakis (dimethylamino)ethylene (TMAE) seeded in a buffer gas to produce a collisional plasma. ${ }^{10}$ TMAE has one of the lowest ionization potentials known today ( 5.36 $\mathrm{eV}){ }^{11}$ Its vapor can be ionized efficiently with photons in the ultraviolet spectrum range, and has been used in UV photon detectors for high energy physics experiments.
Hence, using UV radiation to ionize TMAE is, indeed, an attractive scheme to generate plasmas. Stalder et al. ${ }^{1,15}$ have conducted experiments to study the ionization of TMAE in a helium gas mixture by UV radiation produced by an array of spark gaps. They generated a large volume of collisional TMAE plasma at atmospheric pressure and demonstrated more than $20 \mathrm{db}$ reduction in reflected microwave energy.

In this article, we report on the experimental study of a TMAE plasma produced by a UV laser beam at a wavelength of $193 \mathrm{~nm}$. Our research is motivated by the objective of using a TMAE plasma to reflect, shield, and absorb electromagnetic waves. In order to make these applications successful, the basic TMAE plasma characteristics should be examined carefully. There have been several papers on the absorption cross section and ionization efficiency of TMAE. ${ }^{12-14}$ Recently, the decay process of ionized TMAE in helium at atmospheric pressure has been studied. ${ }^{15}$ In this article we first present a theoretical model of UV ionization of TMAE by the one-photon ionization process, which is necessary to describe the measurement and is essential for obtaining a plasma with a desired spatial density distribution. We then present a basic measurement of TMAE plasma generated by a $193 \mathrm{~nm}$ UV laser beam. The spatial and temporal distributions of electron temperature and density are measured. From the temporal evolution of electron temperature and density the electron-ion recombination coefficient is determined. The spatial density profile along the direction of the laser beam also provides a measurement of the absorption cross section. The microwave scattering measurement results are presented to demonstrate microwave reflection and shielding in a TMAE plasma.

\section{ONE PHOTON IONIZATION MODEL}

It is well known that the impact of a photon on a molecule can cause ionization, if the photon energy $E_{0}=h v$ 
is greater than the ionization threshold of the molecule. We consider a photon flux propagating through a molecular gas, assuming that photoionization is the main absorption process, and omitting the absorption of photons by TMAE ions. The equation describing the photon flux propagation and absorption is derived to be

$$
\frac{\partial \Gamma}{\partial t}=-\frac{c}{n} \frac{\partial \Gamma}{\partial z}-\frac{c}{n} \Gamma\left(n_{m}-n_{e}\right) \sigma_{a},
$$

where $\Gamma$ is the photon flux, and $n_{e}$ and $n_{m}$ are electron density and neutral molecular density, $\sigma_{a}$ is the photon absorption cross section, $c$ is the speed of light in the vacuum, and $n$ is the index of refraction. In our experiment $n=1$, since the laser frequency is much higher than the plasma frequency.

The ionization and electron loss processes can be described by

$$
\frac{\partial n_{e}}{\partial t}=\Gamma\left(n_{m}-n_{e}\right) \sigma_{i}+\alpha_{d} \frac{\partial^{2} n_{e}}{\partial z^{2}}-\alpha_{r} n_{e}^{2}-\alpha_{a} n_{e},
$$

where $\sigma_{i}$ is the ionization cross section; $\alpha_{d}, \alpha_{n}$ and $\alpha_{a}$ are the plasma diffusion coefficient, electron-ion recombination coefficient, and electron attachment frequency to neutral molecules, respectively. The neutral molecules can include impurities having a high electron capture cross section, such as tetramethylurea (TMU) and tetramethyloxamide (TMO) usually present in commercial TMAE ${ }^{16}$ and oxygen that slowly leaks into the system. The solution of Eqs. (1) and (2) yields the spatial and temporal evolution of electron density and photon flux, which are often of interest in pulsed photon ionization experiments and applications. It should be noted that the inclusion of $n_{e} \sigma_{a}$ in Eq. (1) and $n_{e} \sigma_{i}$ in Eq. (2) accounts for the decrease in the density of unionized neutral molecules caused by the ionization which is either the afterglow from the previous photon pulse or comes from the leading edge of the laser pulse. Generally, the leading edge of a photon pulse will see a higher neutral density than that seen by the trailing edge of the pulse. This effect can be very important when the photon flux density is high and the pulse duration is sufficiently long such that the electron density is the same order of magnitude as that of neutral molecules. In our experiment, the percentage ionization is very small $\left(\sim 10^{-3}\right)$, so this effect can be ignored.

In certain situations we are only interested in the steady-state solution, which corresponds to a steady-state UV source illuminating TMAE vapor. We can then set the right hand side of Eq. (1) and (2) equal to zero, and simplify as

$$
\begin{aligned}
& \frac{\partial \Gamma}{\partial z}=-\Gamma\left(n_{m}-n_{e}\right) \sigma_{a}, \\
& \alpha_{d} \frac{\partial^{2} n_{e}}{\partial z^{2}}=-\Gamma\left(n_{m}-n_{e}\right) \sigma_{i}+\alpha_{r} n_{e}^{2} .
\end{aligned}
$$

We have dropped the attachment term on the right hand side of Eq. (2). Here, we are considering an idealized onedimensional half-space in the steady state. When the photon source is turned on at $t=0$, negative ions will accumu-

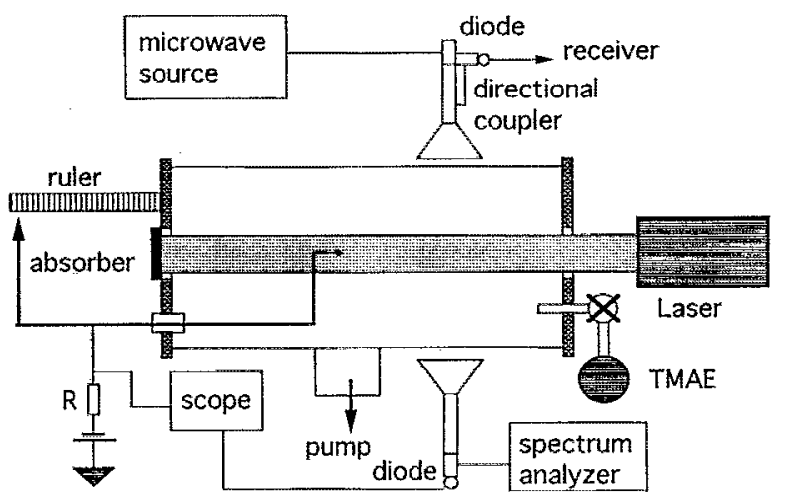

FIG. 1. Schematic of the experimental setup.

late due to attachment. This buildup of negative ions cannot continue indefinitely because of the electron detachment from negative ions. Once a balance is reached, i.e., the electron attachment loss rate is equal to the electron detachment rate from negative ions, there will then be no net loss of electrons. This balance will be achieved first at the position $z=0$, and require longer times for larger values of $z$. From Eqs. (3) and (4) it is clear that if the incident photon flux intensity is known, the electron density profile can be calculated for a given neutral density and other parameters. For a particular application, there is always a preferred electron density profile. We can change the generated density profile to best fit a theoretically optimum one by adjusting the photon flux intensity, neutral density, and plasma diffusion coefficient. The other parameters in Eqs. (3) and (4), namely, photon absorption cross section, ionization cross section, and electron-ion recombination coefficient are measured in our experiment.

\section{EXPERIMENT}

The experimental arrangement is shown in Fig. 1. The vacuum chamber is pumped by diffusion pump, and has a base pressure of $1 \times 10^{-6}$ Torr. The TMAE vapor is introduced through a glass tube from the TMAE container. A Lambda Physik EMG-50E excimer laser is used as the ionization sourcc. The laser beam has a wavelength of 193 $\mathrm{nm}\left(E_{0}=6.4 \mathrm{eV}\right)$, pulse width of $17 \mathrm{~ns}$, maximum energy of $20 \mathrm{~mJ}$, and a beam dimension of $23 \mathrm{~mm} \times 7 \mathrm{~mm}$. The beam is incident into the chamber through one of the two Suprasil windows, which transmit well down to $180 \mathrm{~nm}$. The TMAE plasma shows a very faint glow in the dark. If the system is filled with $\mathrm{N}_{2}$ after the TMAE is introduced, a more intense blue light is visible which is illustrated in Fig. 2 as the horizontal light column in the center of the picture. Since the laser beam is invisible in air which is rich in nitrogen, we believe that the emitted light is produced by the decay from the fast electron excited states of nitrogen molecules. The light coincides with the cross section of the laser beam indicating that the main body of the plasma is located in the long column having the same cross section as the laser beam. The light emission is observed from the suprasil window to the center of the vacuum chamber ( 25 


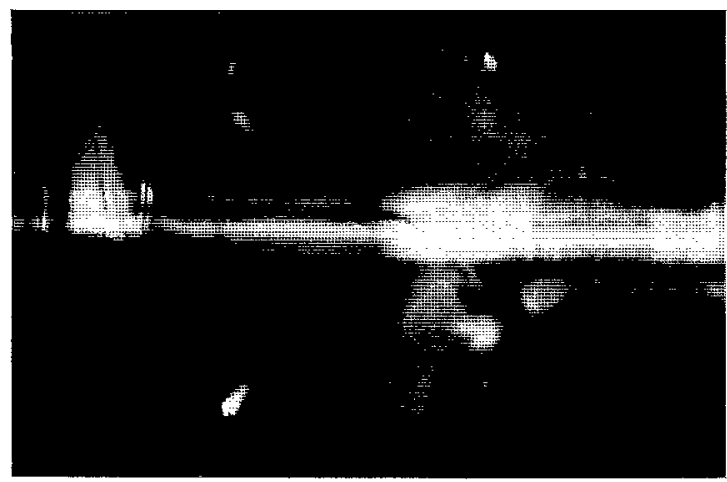

FJG. 2. Plasma column produced in TMAE vapor.

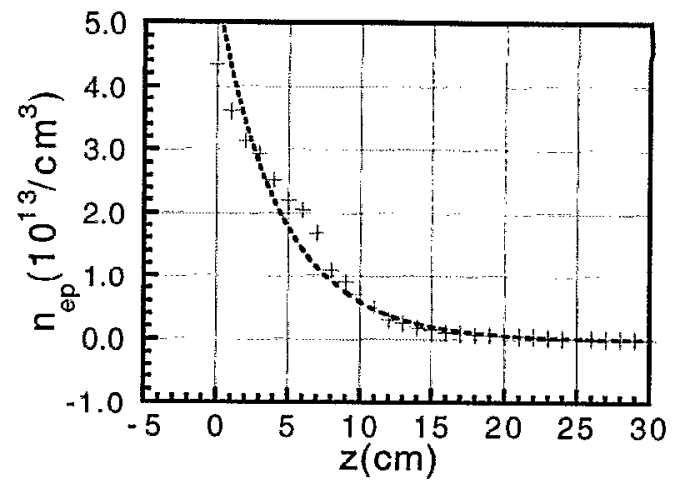

FIG. 3. Electron density profile along the direction of laser beam. cm) when the TMAE pressure is $500 \mathrm{mTorr}$, with the intensity decreasing from the window towards the center. The light column exists all the way to the other side of the chamber, a total length of $50 \mathrm{~cm}$, if the TMAE vapor pressure is reduced to $100 \mathrm{mT}$ Trr or lower.

\section{A. Photon absorption cross-section measurement}

Equations (1) and (2) provide general descriptions of the photon flux in a gas medium where the ionization potential of the molecules is less than the photon energy. If the photon flux is a short pulse of width $\tau$, during that time the electron density will not be affected by diffusion, recombination, or attachment processes, and the percentage of ionization will remain small, i.e., $n_{e} n_{m}$; then Eqs. (1) and (2) can be simplified to

$$
\frac{\partial \Gamma}{\partial z}=-\Gamma n_{m} \sigma_{a},
$$

where $n_{m} \sigma_{a}=k_{a}$ is the absorption coefficient and

$$
n_{e}=\Gamma n_{m} \sigma_{i} \tau \text {. }
$$

The solution of (5) is

$$
n_{e}(\mathbf{Z})=n_{e}(0) \exp \left(-n_{m} \sigma_{d} \mathbf{Z}\right)
$$

where $n_{e}(0)=\Gamma(0) n_{m} \sigma_{i} \tau$. The absorption cross section of TMAE vapor can then be determined after measurement of the electron density spatial profile along the direction of laser propagation.

To measure the electron density spatial distribution, we used a $2.4 \mathrm{~mm}$-diam by 0.1 -mm-thick tantalum disk Langmuir probe, which can be moved axially or transverse to the direction of the laser beam. The electron collecting surfaces of the disk are parallel to the direction of the laser beam to minimize the cross-sectional area in the beam path. The threshold of ablation of metals by a UV laser is the order of $10^{8} \mathrm{~W} / \mathrm{cm}^{2}$, which is at least two orders higher than the laser pulse power in our experimental chamber. ${ }^{17}$

Since tantalum has a work function of $3.76 \mathrm{eV}$ and the photon energy of the $193 \mathrm{~nm}$ laser is $6.4 \mathrm{eV}$, photoelectric emission from the laser beam striking the edge of the tantalum disk has to be considered. We checked this effect experimentally as follows. The probe was positioned in the laser beam close to the window and biased negatively $(-20 \mathrm{~V})$ through a resistor $(1.2 \mathrm{k} \Omega)$. The voltage on the resistor was monitored by connecting it directly to the high impedance input of a digital storage scope which has a maximum sensitivity of $2 \mathrm{mV} /$ division. When the chamber is filled with air from $20 \mathrm{~m}$ Torr to atmospheric pressure, and TMAE is not introduced, the recorded signal is about $5 \mathrm{mV}$ which comes from the radio frequency interference generated by the laser discharge circuitry. No noticeable change is observed whether we block the laser beam or not. Therefore, the maximum current that could be produced by photoelectric emission is $4.2 \mu \mathrm{A}$ which is $2-3$ orders smaller than the ion saturation current measured.

The probe is then biased negatively to measure the peak ion saturation current in the plasma as a function of distance from the window. The current is proportional to electron density generated at the position which is in turn proportional to the photon flux according to $\mathrm{Eq}$. (6). The measurement is conducted at a vapor pressure of 0.5 Torr at a room temperature of $21.5^{\circ} \mathrm{C}$ corresponding to the TMAE molecular density of $n_{m}=1.65 \times 10^{16} / \mathrm{cm}^{3}$. The maximum electron density measured is $5 \times 10^{13} / \mathrm{cm}^{3}$, so $n_{e} \leqslant n_{m}$. Figure 3 shows the peak electron density along the axial direction. The exponential fit of the experimental data gives the absorption coefficient $k_{i}=0.18 / \mathrm{cm}$, a photon mean free path of $l=5.4 \mathrm{~cm}$, and a photon absorption cross section of $\sigma_{a}=1.1 \pm 0.2 \times 10^{17} \mathrm{~cm}^{2}$. This value is about $30 \%$ of that measured by Holroyd et al. ${ }^{14}$ There are many factors that could lead to the difference in results. The linewidth of the laser output is $1 \mathrm{~nm}$ for our laser according to the manufacturer's supplied information. In Holroyd et al.'s experiment, a synchrotron light beam is used, which needs a monochromator to filter out the unwanted spectrum, and no information about the linewidth is given. In our experiment, the photon intensity is measured along the beam path at intervals of $1 \mathrm{~cm}$ for a total length of 30 $\mathrm{cm}$, and an exponential function is then fitted to the data to obtain the absorption coefficient. This should lead to improved accuracy compared to fitting an exponential function to two data points as Holroyd et al. have done. Also, the condensation of TMAE on their windows could have contributed to the photon loss in their experiment. The condensation does not affect our measurement, 


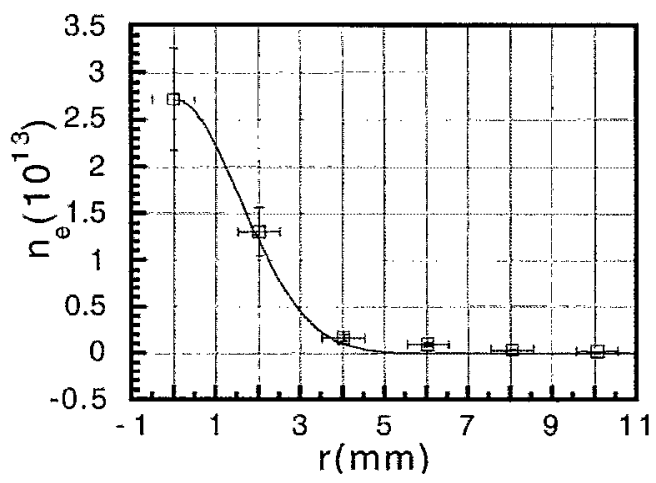

FIG. 4. Radial electron density profile.

since the photon intensity is effectively measured inside the chamber.

To determine the ionization efficiency, we need to know the total number of electrons generated and the laser pulse energy. The total number of electrons generated in a half space is

$$
N_{e}=\int_{0}^{\infty} s n_{e}(0) \exp \left(-n_{m} \sigma_{a} z\right) d z
$$

where the $s$ is the laser beam cross-sectional area. The ionization efficiency is therefore

$$
\eta=\frac{N_{e}}{N_{p}}=\frac{\sigma_{i}}{\sigma_{a}}
$$

where the $N_{p}$ is the total number of photons in the pulse which can be determined from pulse energy measurement. In this experiment, $\eta$ is determined to be 0.52 .

In the above calculation, we have assumed that the electron density is uniform inside the beam and zero outside. The actual electron density radial profile has been examined. A cylindrical laser beam is obtained by passing the rectangular beam through a 7-mm-diam iris. The Langmuir probe is moved radially from the beam center; the peak electron density along the radial direction is shown in Fig. 4. It shows that the electrons are concentrated in the region illuminated by the laser beam, and the radial diffusion is insignificant. This is consistent with the visual observation that the light emission column coincides with the laser beam.

\section{B. Electron temperature measurement}

When a photon having an energy $E_{0}$ ionizes a TMAE molecule, the free electron is expected to have a kinetic energy of $E_{0}-E_{p}$, where $E_{p}$ is the ionization potential. Hence, right after the laser pulse, the electron velocity distribution is highly non-Maxwellian. The Langmuir probe temperature measurement will deviate from that predicted by probe theory for a non-Maxwellian plasma. But the TMAE molecule (which has a formula weight of 200) has a large electron collision cross section. If we estimate the electron neutral collision frequency $v$ to be $\sim 10^{9} p$, where $p$ is the pressure in Torr, a reasonable estimation of the re-

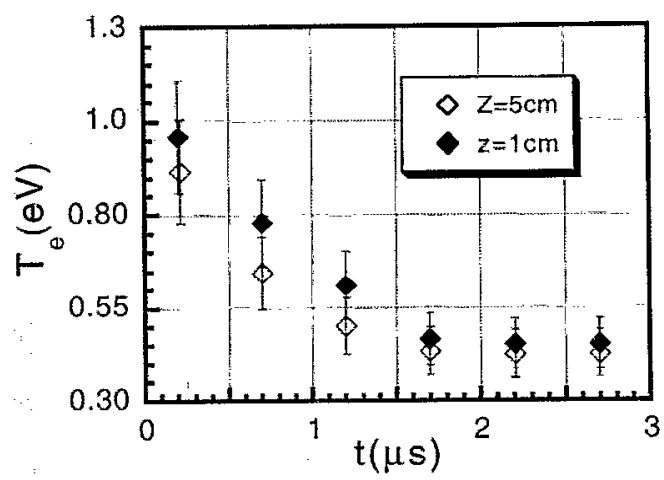

FIG. 5. Electron temperature vs time.

laxation time is on the order of $10 / v$, i.e., $10 \mathrm{~ns}$. Therefore, we can consider that the electron density distribution function will relax to a Maxwellian almost immediately after the UV pulse. Figure 5 is an electron temperature plot versus time at $z=1 \mathrm{~cm}$ and $z=5 \mathrm{~cm}$, where $t=0$ corresponds to the leading edge of the laser pulse. The electron temperatures measured at $t=0.2 \mu \mathrm{s}$ are $1.0 \mathrm{eV}$ for $z=1 \mathrm{~cm}$ and $0.92 \mathrm{eV}$ for $z=5 \mathrm{~cm}$.

\section{Electron-ion recombination coefficient measurement}

The plasma decay process can be described by Eq. (2) by setting $\Gamma=0$

$$
\frac{\partial n_{e}}{\partial t}=\alpha_{d} \frac{\partial^{2} n_{e}}{\partial z^{2}}-\alpha_{r} n_{e}^{2}-\alpha_{a} n_{e}
$$

TMAE has a molecular weight of 200 . The ambipolar diffusion coefficient of TMAE is estimated to be $1.65 \times 10^{4}$ $\mathrm{cm}^{2} / \mathrm{s}$ and the plasma decay time constant is estimated to be $0.59 \times 10^{-2} \mathrm{~s}$ at room temperature. Therefore, the diffusion can be neglected on a time scale shorter than a ms. In TMAE vapor, the impurity TMU has about the same order of magnitude of electron capture cross section as oxygen. ${ }^{16}$ The impurity TMO clectron capture cross section is several times larger. ${ }^{16}$ Rewick et al.'s analysis ${ }^{16}$ of commercial TMAE shows that the concentration of TMO is $0.04 \%$ and that of TMU is $0.31 \%$. We can, therefore, estimate the effective attachment rate using the available data on electron attachment to oxygen. ${ }^{18}$ The electron attachment frequency to neutral oxygen molecules is $v_{a}=2$ $\times 10^{-13} n_{\mathrm{im}}$ for an electron attachment cross section of $3 \times 10^{-21} \mathrm{~cm}^{2}$, where $n_{\mathrm{im}}$ is the impurity density of molecules which have a large electron capture cross section. The oxygen in our vacuum system is calculated from the leak rate of our vacuum system to be less than $0.2 \%$. Assuming the impurities total $1 \%$ in the system, the corresponding decay time constant is calculated to be on the order of $10 \mathrm{~ms}$. So the initial decay of electron density is dominated by electron-ion recombination, and it obeys the well-known simple equation 


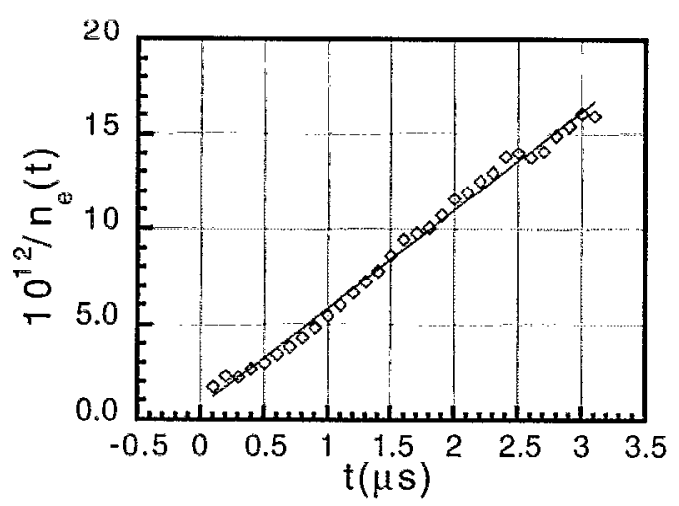

FIG. 6. Inverse of electron density vs time.

$$
n(t)=\frac{n_{0}}{n_{0} \alpha_{r} t+1},
$$

where $n_{0}$ is the initial electron density.

The initial electron decay is measured with a Langmuir probe. Figure 6 shows the inverse of electron density versus time. The good fit of the experimental data to a linear function shows that the decay is, indeed, recombination. The recombination coefficient is determined to be $5.4 \pm 0.5$ $\times 10^{-6} \mathrm{~cm}^{3} / \mathrm{s}$.

\section{Microwave scattering measurement}

The microwave reflection experimental setup is shown in Fig. 1. The microwave beam is incident on the plasma column in the direction transverse to the laser beam. The center of the antenna horn is $18 \mathrm{~cm}$ from the end plate. The antenna is a $7.5 \mathrm{~cm} \times 9.5 \mathrm{~cm}$ rectangular horn placed just outside of the glass chamber. The distance from the antenna to the plasma column is about $7 \mathrm{~cm}$. The microwave power $(10 \mathrm{~mW})$ is incident on the narrow side $(0.7 \mathrm{~cm})$ of the plasma column. The microwave frequency is adjusted to allow the reflected microwave power from the chamber walls to be in phase with the peak reflection from the plasma column, and is experimentally determined to be 8.7 GHz. The peak electron density in the region in front of the antenna is measured by a Langmuir probe to be the order of $10^{13} \mathrm{~cm}^{-3}$ at a pressure of 250 mTorr.

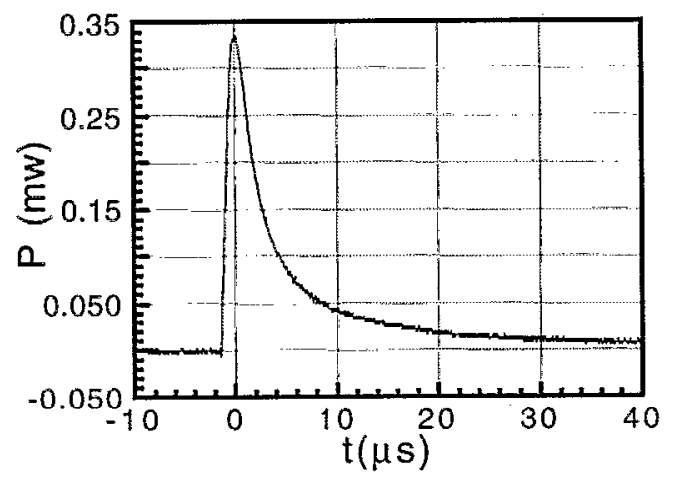

FIG. 7. Microwave power reflected from TMAE plasma column.

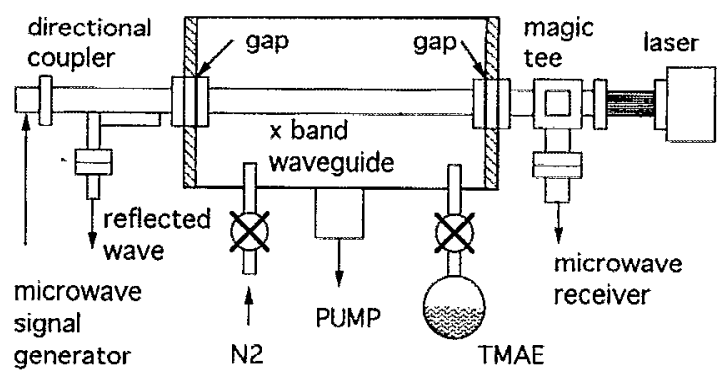

FIG. 8. Schematic of wave scattering in TMAE plasma filled waveguide.

The reflected wave is measured by a diode detector through a directional coupler, which is shown in Fig. 7. The peak reflected power is $0.33 \mathrm{~mW}$. For comparison, the $8.7 \mathrm{GHz}$ microwave signal reflected from an $X$-band waveguide $(25 \mathrm{~mm} \times 13 \mathrm{~mm})$ placed at the same position as the plasma column is measured to be $0.45 \mathrm{~mW}$. The result demonstrates that reflection due to the TMAE plasma can be very effective. In the future we plan to produce a sheet ultraviolet beam to create a high density gradient TMAE plasma sheet with a width several times the wavelength of the microwaves. The plasma sheet should be able to effectively reflect microwaves. If the UV laser beam is optically rotated, a rotating mirror reflector is produced which would be attractive for radar scanning applications at microwave or millimeter wavelengths.

Figure 8 shows another setup for the microwave
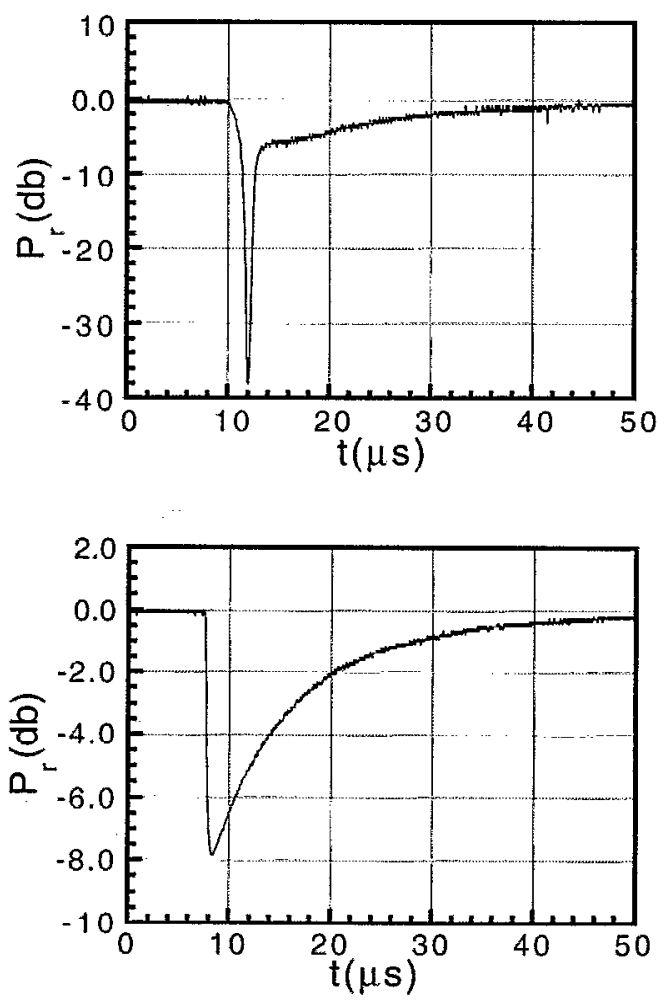

FIG. 9. Microwave transmission through a waveguide filled with a transient TMAE plasma generated by an UV pulse: (a) high initial density, (b) lower initial density. 


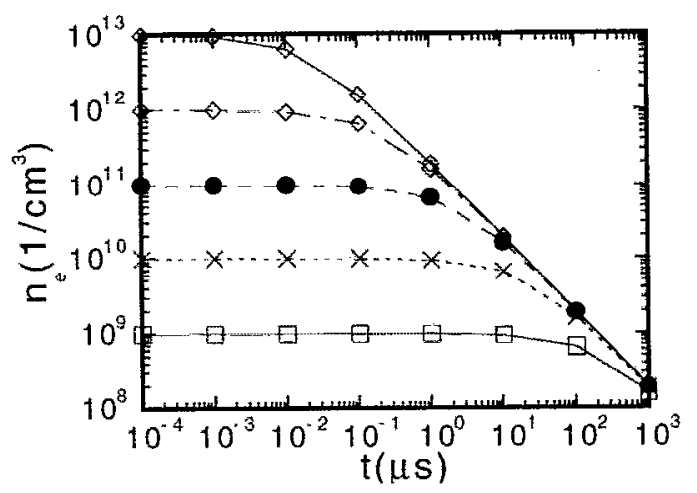

FIG. 10. Electron density decay in TMAE plasma by recombination for different initial densities.

plasma interaction experiment to demonstrate wave shielding effects. In this arrangement, the laser beam is injected through a magic-tee into an $X$-band waveguide. The magictee also couples microwaves into the same waveguide where the plasma generated by the laser fills the whole waveguide. Figure 9 (a) shows that the received signal level drops by $38 \mathrm{~dB}$ indicating a total cutoff due to the high electron density produced by the $4 \mathrm{~mJ}$ laser pulse; the signal quickly recovers to $\mathrm{a}-8 \mathrm{~dB}$ level in less than $2 \mu \mathrm{s}$ and then decays at a much slower rate. Figure 9(b) illustrates an $8 \mathrm{~dB}$ signal level reduction when the laser pulse energy is reduced to an estimated value of $0.02 \mathrm{~mJ}$. Since the initial density generated is much lower in this case, the signal lcvel recovers slowly indicating a slow electron density decay. These features are in good agreement with the nature of recombination loss in the TMAE plasma.

To get a quantitative idea of electron recombination loss in the TMAE plasma, we plot the electron density decay given by Eq. (11) with the measured electron-ion recombination coefficient in Fig. 10, for five different initial electron densities. The high initial electron density of $10^{13}$ drops to $1 / e$ of its initial value in about $0.05 \mu \mathrm{s}$; and the lower initial density of $10^{11}$ decays to $1 / e$ of its initial value in about $10 \mu \mathrm{s}$. If one uses repetitive pulses to sustain the electron density above certain value, e.g., $1 / e$ of the initial density, it can be shown that the power required is proportional to the square of the initial electron density. Therefore, it is more economical to use the afterglow TMAE plasma for the purpose of wave absorption and shielding in the lower frequency range, e.g., $3 \mathrm{GHz}$ and below, since it requires much less power. On the other hand, the TMAE plasma is more suitable for rotating mirror applications at high frequencies, e.g., $10 \mathrm{GHz}$ and above, since the fast electron density decay at high initial densities can be utilized to rotate the reflecting plasma sheet very rapidly.

\section{SUMMARY}

We have investigated TMAE plasma generated by a UV laser beam. The photon absorption cross section for $193 \mathrm{~nm}$ UV radiation in TMAE has been determined from electron density profile measurements. The experiment shows that the plasma decay in TMAE is primarily via electron-ion recombination. The electron-ion recombination coefficient has been measured. Effective microwave refiection and shielding effects are demonstrated by scattering measurements. A set of two partial differential equations arc derived to model the one photon ionization process in a low ionization potential gas. The experimental results show that this model is a good description of the TMAE plasma generation by the UV (193 nm) laser. With this model and given parameters, the numerical solution can help in the design and control of TMAE or other low ionization energy gas plasmas which have attractive properties for applications of microwave reflection, shielding, and absorption.

\section{ACKNOWLEDGMENTS}

We wish to thank Professor James C. Weisshaar for the use of his laser equipment, and Robert $\mathbf{J}$. Noll, Owen Eldridge, and Binshen Meng for valuable technical help. This work was supported by AFOSR Grant 89-0353B.

${ }^{1}$ K. R. Stalder, R. J. Vidmar, and D. J. Eckstrom, J. Appl. Phys. 72, 5089 (1992)

${ }^{2}$ W. W. Destler, J. E. DeGrange, H. H. Fleischmann, J. Rodgers, and Z. Segalov, J. Appl. Phys. 69, 6313 (1991).

${ }^{3}$ S. P. Kuo and Y. S. Zhang, Phys. Fluids B 2, 667 (1990).

${ }^{4}$ J. E. Scharer, O, C. Eldridge, S. F. Chang, Y. S. Zhang, M. Bettenhausen, and N. T. Lam, IEEE Trans. Plasma Sci. (to be published).

${ }^{5}$ P, A. Kossey, R. A. Shanny, and E. C. Field, AGARD Conference Proceedings, Bergen, Norway, May 28-31, 1990, No. 485, paper 17A. ${ }^{6}$ S. P. Kuo, Y. S. Zhang, R. J. Barker, and P. Kossey, AGARD Conference Proceedings, Bergen, Norway, May 28-31, 1990, NO. 485, paper $18 \mathrm{~B}$.

${ }^{7}$ W. M. Manheimer, IEEE Trans. Plasma Sci. 19, 1228 (1991).

${ }^{8}$ A. E. Robson, R. L. Morgan, and R. A. Meger (unpublished).

${ }^{9}$ R. J. Vidmar, IEEE Trans. Plasma Sci. 18, 733 (1990),

${ }^{10}$ J. R. Woodworth: T. A. Green, and Frost, J. Appl. Phys. 57 (1985).

${ }^{11}$ Y. Nakato, M. Ozaki, A. Egawa, and H. Tsubomura, Chem. Phys. Lett. 15 June, 615 (1971).

${ }^{12}$ D. F. Anderson, IEEE Trans. Nucl. Sci. NS-28, 842 (1981).

${ }^{13}$ G. Melchart, G. Charpak, and F. Sauli, IEEE Trans. Nucl. Sci. (1990),

${ }^{14}$ R. A. Holroyd, I. M. Preses, C. L. Woody, and R. A. Johnson, Nucl. Instrum. Methods in Phys. Research A 261, 440 (1987).

${ }^{15}$ K. R. Stalder and D. J. Eckstrom, J. Appl. Phys. 72, (1992).

${ }^{16}$ R. T. Rewick and M. L. Schumacher, Analyt. Chem. 60, 2095 (1988).

${ }^{77}$ D. V. Gaidarenko, A. G. Leonov, and I. V. Novobrantsev, Sov. Tech. Phys. Lett. 15, 112 (1989).

${ }^{18}$ S. C. Brown, Basic Data of Plasma Physics (M.I.T., Cambridge Press, 1966), p. 199. 